(C) 2018 IEEE. Personal use of this material is permitted. Permission from IEEE must be obtained for all other uses, in any current or future media, including reprinting/republishing this material for advertising or promotional purposes, creating new collective works, for resale or redistribution to servers or lists, or reuse of any copyrighted component of this work in other works. Final, published version is available at https://doi.org/10.1109/BIOROB.2018.8488123.

\title{
Validation of Online Intrinsic and Reflexive Joint Impedance Estimates using Correlation with EMG Measurements
}

\author{
Ronald C. van 't Veld ${ }^{1}$, Alfred C. Schouten ${ }^{1,2}$, Herman van der Kooij ${ }^{1,2}$ and Edwin H. F. van Asseldonk ${ }^{1}$
}

\begin{abstract}
Biofeedback of online system identification estimates of intrinsic and reflexive joint impedance can be used by able-bodied subjects to voluntarily modulate their reflexive impedance independent of the intrinsic contribution. Similar to EMG-based paradigms, this could potentially be used to reduce muscle hyperreflexia in people with spasticity by facilitating spinal neuroplasticity. However, it remains unanswered if spastic participants are able to use this specific feedback to modulate their reflexes. We show, while subjects were free to co-contract, that the system identification measures have a large linear association with independently measured and processed EMG measures. The impedance estimates were obtained using an existing algorithm with incremental improvements to increase general applicability and decrease bias on the identified parameters in both simulation an experimental data. The correlation with EMG-based measures demonstrates the validity of the use of joint impedance measures within a training paradigm to reduce hyperreflexia. This could potentially improve participant comfort, increase applicability across joints, target hyperreflexia at joint level and generate faster training effects.
\end{abstract}

\section{INTRODUCTION}

Spasticity is a common syndrome caused by various brain and neural injuries, such as spinal cord injury or cerebral palsy. Spasticity, clinically defined as a velocitydependent resistance of a muscle to stretch [1], can severely impair walking ability and functional independence. It is mainly caused by an exaggerated muscle stretch reflex, i.e. muscle hyperreflexia [2]. Concurrent with this hyperreflexia, increased background activity is reported in spastic limbs [3].

To improve the functional independence, methods are sought to reduce muscle hyperreflexia. Recently, feedback on H-reflex amplitude, evoked with electrical stimuli, was used to facilitate spinal neuroplasticity reducing hyperreflexia [4]. Unfortunately, electrical stimuli can be uncomfortable and can only be applied to a limited amount of muscles. A similar protocol using feedback on reflex amplitude evoked with mechanical stimuli has also proven successful [5]. Both EMGbased paradigms showed that hyperreflexia can be downconditioned without altering background muscle activation, although at least 4-6 training sessions were required.

Alongside EMG-based feedback, online estimates of reflexive joint impedance, obtained using system identification [6], could also be used to provide feedback on hyperreflexia. Joint impedance consists of three contributions [7]:

\footnotetext{
*This work was supported by the Netherlands Organisation for Scientific Research (NWO), domain Applied and Engineering Sciences under project number 14903

${ }^{1}$ Department of Biomechanical Engineering, University of Twente, Enschede, The Netherlands

${ }^{2}$ Department of Biomechanical Engineering, Delft University of Technology, Delft, The Netherlands
}

1) an intrinsic contribution due to limb inertia and viscoelastic properties of muscle fibers and tissues in rest;

2) an intrinsic contribution due to properties of activated muscle fibers;

3) a reflexive contribution due to neural reflex activity induced by changes in the muscle, such as the shortlatency stretch reflex.

Using feedback on reflexive joint impedance, it was shown that able-bodied participants can voluntarily modulate reflexive activity without altering intrinsic impedance [6].

The protocol based on system identification has multiple advantages compared with the EMG-based paradigms. First, reflex activity modulation is targeted at joint level, which could help to better facilitate functional improvements [8]. Second, the use of electrical stimulation is avoided, which improves participant comfort and increases general applicability to joints across the body. Third, significant training effects were already attained after 2, instead of 4-6, sessions.

Currently, only two articles describe the online separation of intrinsic and reflexive contributions to joint impedance, using a powerful hydraulic actuator to perturb the ankle. First, an experimental study showed the voluntary modulation of reflexive activity, using an initial version of the algorithm [6]. Second, a simulation study investigated the algorithm characteristics, using an updated version [9]. All experimental and simulation results were obtained with a specific combination of hard- and software and able-bodied participants. As such, challenges remain in terms of general applicability to other hard- and software combinations and whether spastic participants can voluntarily modulate reflexive joint impedance to consequently down-regulate their hyperreflexia.

The main goal of this paper is to validate if the system identification targets the same measures as the EMG-based protocols in an experimental setting, as a first step towards down-regulation of hyperreflexia in spastic participants. Previous results show that identified reflexive impedance increases with reflex EMG for constant background activity [6]. However, the response to varying background activity is also important as it is increased in spastic limbs. Moreover, the association between intrinsic impedance and background activity is unknown. We expect the following associations:

1) identified intrinsic stiffness is correlated with background EMG activity, i.e. intrinsic joint stiffness is correlated with muscle co-contraction;

2) identified reflexive gain is correlated with reflex EMG activity, i.e. mechanical reflex activity is correlated with neural reflex activity. 
Before the system identification is validated, incremental improvements on top of the method in [9] are developed and tested in simulation. The improvements include: revised algorithm filters, differentiators and simulation configuration, and a reshaped perturbation signal. These are aimed at reducing the gap between simulation and experiment, and improving general applicability and quality of the method. Specifically, this considers limited actuator bandwidth and low signal-to-noise ratios (SNRs). Moreover, the system identification is adapted to our experimental setup in terms of sampling frequency and direct velocity measurements.

\section{ONLINE JOINT IMPEDANCE ESTIMATION}

The online impedance estimation consists of three components: the (perturbation) signal, the (simulation) model and the (identification) algorithm. The signal excites the system, the model in simulation and the participant during experiments. The algorithm identifies the impedance parameters given the perturbation signal and system response. The proposed modifications are split into changes with respect to general applicability (conceptual) and the specific experimental setting used (experimental).

\section{A. Original Signal, Model \& Algorithm}

The original signal, model and algorithm are elaborately described in [9], see its nine step guide in Section II-D for the exact implementation of the algorithm. The model used is presented in Fig. 1 and is simulated using a fixed-step solver with $1 \mathrm{~ms}$ increment. White noise was added to the position and torque signals in simulation to obtain an SNR of 70 and $40 \mathrm{~dB}$, respectively.

The algorithm first estimates the intrinsic impedance parameters, inertia $I$, viscosity $B$ and stiffness $K$ by finding the least-squares solution based on the cross-correlations between position and its derivatives, and torque. To use this method, any voluntary and reflex contributions should be eliminated. To remove voluntary torque contributions, a $0.033 \mathrm{~Hz}$ high-pass filter is used. To remove the reflex contributions, it is assumed that the reflexive impulse response function (IRF) between reflexive torque and halfwave rectified velocity is nonzero between 40 and $400 \mathrm{~ms}$.

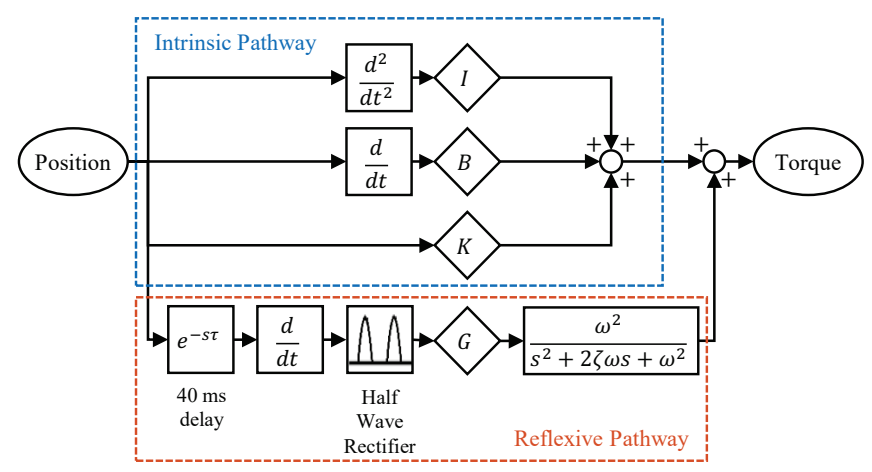

Fig. 1. Joint impedance model with the intrinsic (inertia $I$, viscosity $B$ and stiffness $K$ ) and reflexive (gain $G$ ) parameters [9]
Given these assumptions, a dedicated pulse-step perturbation signal is used to eliminate the reflex. This input signal randomly switches between pulses, defined as a $40 \mathrm{~ms}$ wide block pulse, and steps, defined as a $460 \mathrm{~ms}$ wide block pulse. As such, the cross-correlation between position and half-wave rectified velocity of the signal equals zero between lags of 40 and $460 \mathrm{~ms}$, mathematically eliminating the contribution of the reflex IRF.

After the intrinsic parameters are identified, the corresponding intrinsic torque is estimated and subtracted from the measured torque to obtain the reflexive torque. The reflexive IRF is estimated non-parametrically using the least-squares method, based on reflexive torque and half-wave rectified velocity, and summed to calculate the reflexive gain, $G$.

\section{B. Signal Modifications}

1) Conceptual Modification: The theoretically designed perturbation complies with the requirement that the crosscorrelation between position and half-wave rectified velocity be zero between lags of 40 and $400 \mathrm{~ms}$. However, actuators with a finite bandwidth, effectively filtering the signal, fail to comply, see Fig. 2. The actuator of our specific experimental setup could not achieve a bandwidth higher than $30 \mathrm{~Hz}$ for this perturbation. The finite bandwidth also smooths the correlation function making the block-shaped features less distinct. To mitigate this issue, a 5th-order polynomial with a length of $24 \mathrm{~ms}$ is used to shape the signal in order to meet the correlation requirement, see Fig. 2.

\section{Model Modifications}

1) Conceptual Modifications: The model should represent reality as close as possible to reduce the gap between simulation and experiment. Therefore, we use actual velocity and acceleration during simulations, instead of their approximations based on numerically differentiating position, see Fig. 1. Similarly, a variable-step, instead of a fixed-step, solver is selected as it is better suited to simulate continuous models. To further mimic a realistic setup, anti-aliasing filters at $90 \%$ of the Nyquist frequency are used before sampling.

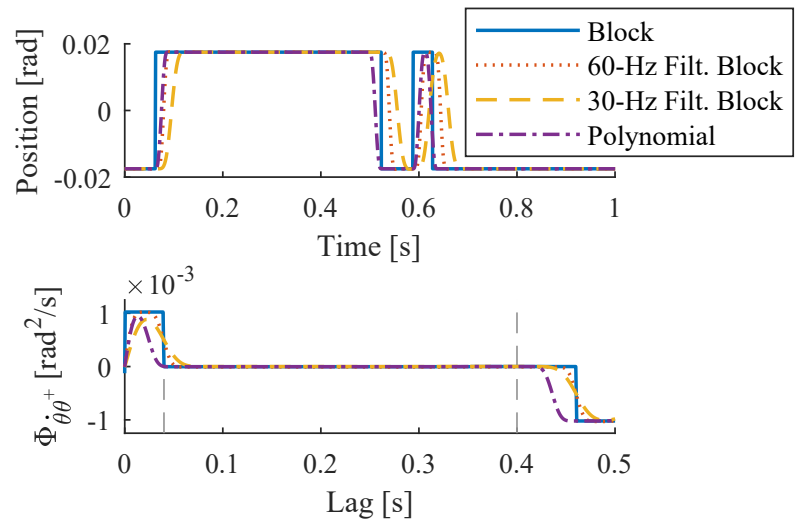

Fig. 2. (Top) Time domain visualization of the block, $60 \mathrm{~Hz}-$ and $30 \mathrm{~Hz}-$ filtered block and polynomial input signals; (Bottom) Cross-correlations between position and half-wave rectified velocity of input signals, required to be zero between lags of 40 and $400 \mathrm{~ms}$ 
2) Experimental Modifications: The sampling frequency of the experimental device used is $2048 \mathrm{~Hz}$ instead of $1000 \mathrm{~Hz}$ in the original paper [9]. Besides, the high SNR values of 70 and $40 \mathrm{~dB}$ for position and torque were replaced by 52.7 and $24.1 \mathrm{~dB}$ based on a dedicated experiment with our device.

\section{Algorithm Modifications}

1) Conceptual Modifications: The original algorithm is implemented using analog filters within a Simulink/xPC framework (Mathworks, Inc., Natick, MA, USA). To improve the applicability in other frameworks, which cannot easily solve ordinary differential equations, a digital, discrete version of the algorithm is used. Therefore, the analog Bessel filters, which lack a digital equivalent, are replaced with digital Butterworth filters with the same order and cutoff frequencies. Moreover, all 1st-order backward difference approximations are replaced with 4th-order approximations, to closer match the model modification from using the actual velocity and acceleration. To complement this change all inputs are low-pass filtered at $100 \mathrm{~Hz}$ to attenuate the noise amplification due to the higher order numerical differentiation. Given the limited bandwidth of the perturbation signal, no relevant dynamics should appear above this frequency.

2) Experimental Modifications: All algorithm features depending on the sampling frequency are adapted to retain their characteristics at the new $2048 \mathrm{~Hz}$ sampling frequency. Furthermore, the velocity measurement available in the device (SinCos encoder, $37.7 \mathrm{~dB}$ SNR) is used instead of differentiating measured position.

\section{Methods}

The results were obtained using a simulation and an experimental study. The goal of the simulation was to test the effect of all conceptual and experimental modifications. The goal of the experiment was to validate the system identification algorithm with respect to the EMG measurements.

\section{A. Simulation Study}

As in [9], all simulations were executed using Simulink. The simulations ran for $240 \mathrm{~s}$ and the first $30 \mathrm{~s}$ of the parameter estimations were ignored to avoid errors due to simulation transients. The simulation runs were performed for six modification combinations, see Table I.

All these combinations were assessed on accuracy, variability and risetime, using the same measures as in [9]. The variance accounted for, \%VAF calculated for a set of true parameter values, $x$, and their estimates, $\hat{x}$, as

$$
\% \mathrm{VAF}=\left[1-\frac{\operatorname{var}(\hat{x}-x)}{\operatorname{var}(x)}\right] \cdot 100 \%
$$

was used to assess identification accuracy for 100 simulation runs for three simulated conditions:

1) a random intrinsic stiffness $(K)$, between $0-200$ $\mathrm{Nm} / \mathrm{rad}$, with a fixed reflexive gain $(G)$ of $10 \mathrm{Nm} / \mathrm{rad} / \mathrm{s}$;

2) a random $G$, between $0-20 \mathrm{Nm} / \mathrm{rad} / \mathrm{s}$, with a fixed $K$ of $100 \mathrm{Nm} / \mathrm{rad}$

3) both a random $K$, between $0-200 \mathrm{Nm} / \mathrm{rad}$, and a random $G$, between $0-20 \mathrm{Nm} / \mathrm{rad} / \mathrm{s}$.
TABLE I

OVERVIEW OF THE SIX MODIFICATION COMBINATIONS USED: $\mathrm{O}=$ ORIGINAL $; \mathrm{C}=$ CONCEPTUAL $; \mathrm{E}=$ EXPERIMENTAL

\begin{tabular}{cc|ccc} 
& \multicolumn{3}{|c}{ Algorithm } \\
& O & C & CE \\
\hline$\frac{\mathrm{O}}{\mathrm{E}}$ & $\mathrm{O}$ & OO & OC & \\
C & CO & CC & \\
CE & CEO & & CECE
\end{tabular}

Additionally, a single simulation run with a fixed $K$ of $100 \mathrm{Nm} / \mathrm{rad}$ and a $G$ of $10 \mathrm{Nm} / \mathrm{rad} / \mathrm{s}$ was used to assess variability. While another simulation run with a jump in $K$ from 50 to $150 \mathrm{Nm} / \mathrm{rad}$ at $100 \mathrm{~s}$ and a jump in $G$ from 5 to $15 \mathrm{Nm} / \mathrm{rad} / \mathrm{s}$ was used to assess the algorithm risetime. The risetime was defined as the time the identified parameters require to go from $10 \%$ to $90 \%$ of the modeled jump. All other parameters were fixed with an intrinsic viscosity $(B)$ of $0.63 \mathrm{Nm} / \mathrm{rad} / \mathrm{s}$ and inertia $(I)$ of $0.0137 \mathrm{Nm} / \mathrm{rad} / \mathrm{s}^{2}$ and a reflexive frequency $(\omega)$ of $21 \mathrm{rad} / \mathrm{s}$ and damping $(z)$ of 0.8 .

\section{B. Experimental Study}

The experimental study was executed in similar fashion to Experiment 1 of [6]. Three male able-bodied subjects, aged 23-27, participated in the study.

1) Apparatus: Subjects were seated with their right foot attached to an actuator (MOOG, Nieuw-Vennep, the Netherlands) using a rigid footplate and Velcro straps, see Fig. 3. While in [6], subjects laid supine with their left foot attached using a custom-fitted fiberglass boot. The system applied one degree of freedom perturbations in the sagittal plane around the ankle joint, using a separate dedicated real-time computer for control. The ankle and actuator axis of rotations were visually aligned before the start of the experiment.

The ankle position, velocity and torque were measured at $2048 \mathrm{~Hz}$, all positive in dorsiflexion direction. Moreover, the muscle activity of the Soleus (SOL), Tibialis Anterior (TA), Gastrocnemius Lateralis (GL) and Gastrocnemius Medialis (GM) were measured at $2048 \mathrm{~Hz}$ using the Porti (TMSi, Oldenzaal, the Netherlands). The EMG electrodes were placed according to the SENIAM guidelines [10]. Matlab (Mathworks, Inc., Natick, MA, USA) was used to process all data and provide the user with biofeedback at a rate of

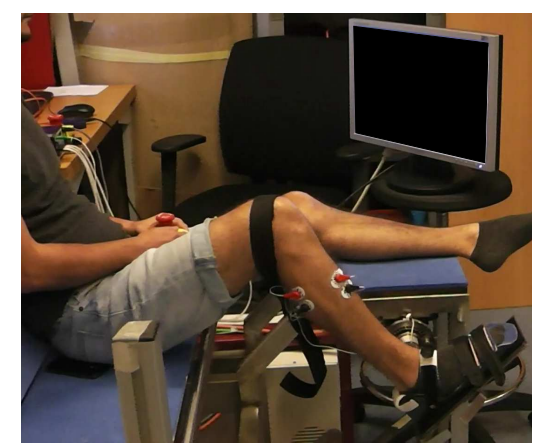

Fig. 3. Experimental setup: subjects were seated on an adjustable chair such that both ankle and knee angles were about $90^{\circ}$ with the knee supported using an additional Velcro strap 


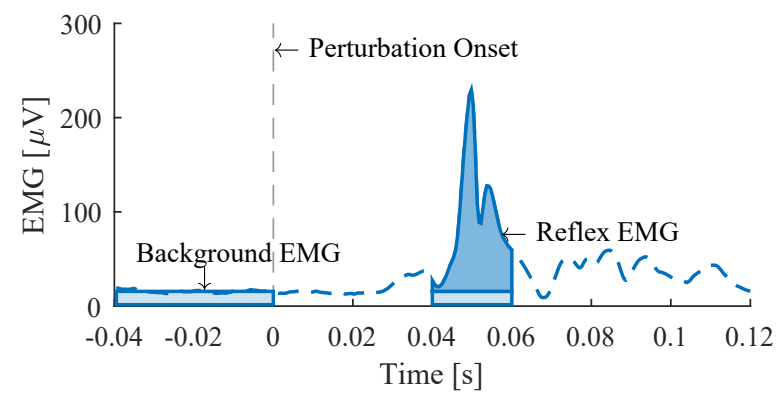

Fig. 4. Background EMG activity was calculated as the average activity between 0 and $40 \mathrm{~ms}$ before the dorsiflexing stretch onset. Reflex activity was calculated as the area under the curve minus the background activity between 40 and $60 \mathrm{~ms}$ after perturbation onset

20-30 Hz. The 2D feedback screen provided a torque target by means of an upper and lower bound on the y-axis and feedback on reflexive gain or intrinsic stiffness on the $\mathrm{x}$-axis.

2) Procedure: The experiment aimed at training subjects to modulate intrinsic stiffness or reflex activity at two torque levels, 0 and $-5 \mathrm{Nm}$. No specific instruction on modulation strategies were given and co-contraction was permitted. Perturbations were applied continuously for four blocks of 1012.5 minutes with a 2.5-5 minute break in between. Feedback was provided on torque and intrinsic stiffness or reflexive gain. Subjects were requested to hold their state/behavior for a $60 \mathrm{~s}$ period for each novel state, i.e. combination of torque and intrinsic stiffness or reflexive gain, found.

3) Data Analysis: All EMG data were first high-pass filtered at $20 \mathrm{~Hz}$ and rectified. Next, all $60 \mathrm{~s}$ hold periods were retrieved and an estimate of intrinsic stiffness and reflexive gain was obtained for each period by averaging over 60 s. Similarly, the approximate 100 EMG reflex responses were ensemble averaged using the dorsiflexing perturbation onset for alignment. Afterwards, background and reflex EMG measures were calculated in similar fashion to [4], see Fig. 4. Given that the TA is stretched in plantarflexion direction, the reflex measure for the TA muscle was calculated using the plantarflexing perturbation onset as reference.

4) Statistical Analysis: The data analysis provided paired data points for the system identification and EMG measures for each hold period performed by every subject. Pearson's correlation coefficient, $r$, was used to analyze the amount of linear association between the measures. However, all subjects had a unique slope and intercept concerning this association. Therefore, the Z-score was used as normalization for every measure and each subject, see Fig. 5. The robustness of the calculated correlation coefficients to any nonlinearities or outliers in the dataset was investigated by calculating the 95\% confidence interval (CI). A non-parametric bootstrap procedure, using the bias corrected and accelerated method to construct the CI [11], was implemented in R [12].

\section{Results}

The main result discussed is the amount of linear association between online estimates of intrinsic and reflexive joint impedance and their respective EMG measures. These results

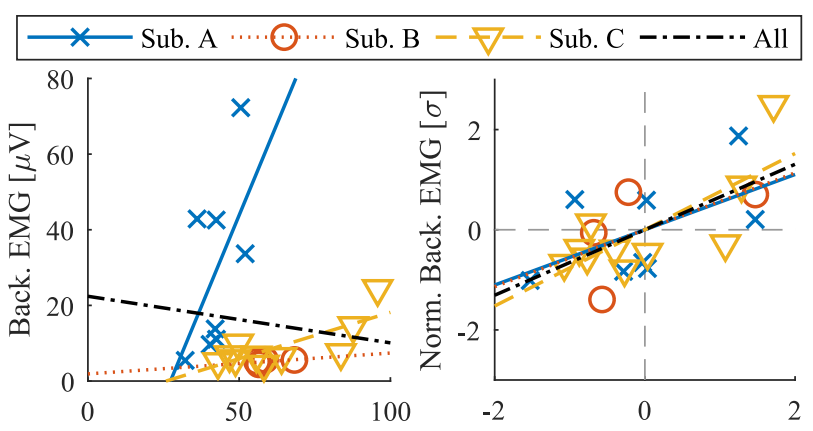

Intrinsic Stiffness [Nm/rad] Norm. Intrinsic Stiffness $[\sigma]$

Fig. 5. (Left) Absolute and (Right) Normalized correlation analysis for all subjects; Data has to be normalized such that the overall slope is representative for every individual subject

are supported with a simulation study to gain insight into the characteristics of the algorithm, the applied modifications and the effect of the experimental setting used.

\section{A. Signal-Model-Algorithm Modifications}

First, the results for all six signal-model and algorithm combinations, see Table II, show similar characteristics for the original and its replication (OO condition). For example, all deviations in the mean of the parameters are smaller than half the standard deviation.

Second, the conceptual modifications are investigated with the $\mathrm{OO}, \mathrm{OC}, \mathrm{CO}$ and $\mathrm{CC}$ conditions. The lowest bias on parameters is obtained when the signal-model and algorithm settings match with each other, i.e. the $\mathrm{OO}$ and $\mathrm{CC}$ conditions. Thus, the use of the higher-order backwards difference approximators and $100 \mathrm{~Hz}$ low-pass filters is beneficial when using the model with more realistic assumptions ( $\mathrm{CC}$ condition). Besides, the limited actuator performance (maximum velocity/acceleration and bandwidth) causes an increase in variability of the identified intrinsic stiffness and reflexive gain, see $\mathrm{CO}$ and $\mathrm{CC}$ conditions, due to the smoothed correlations as discussed for Fig. 2 in Section III-B.

Third, the experimental modifications are investigated with the CEO and CECE conditions. The CEO condition shows that the original method cannot cope with the decreased SNR on the inputs, which biases the auto-correlations used to identify the intrinsic parameters. Consequently, all identified parameters are significantly biased compared to the other conditions. Note, this bias also causes the error in the estimation of the risetime. Contrarily, due to the direct velocity measurements and the $100 \mathrm{~Hz}$ low-pass filters, the results of the CECE condition are satisfactory without any large biases, variability or risetimes compared with the $\mathrm{OO}$ condition, regarded as baseline.

\section{B. Comparison Simulation and Experimental Study}

The large differences between the O- and CE-algorithm for the CE-model condition should be confirmed by the experimental results. The identified intrinsic stiffnesses strongly co-vary with a similar offset between the algorithms in both studies, Fig. 6. Moreover, the CE-algorithm estimates are 
TABLE II

ORIGINALLY PUBLISHED VALUES AND SIMULATION RESULTS FOR ALL SIX MODEL/ALGORITHM COMBINATIONS CONCERNING THE ACCURACY, $\% \operatorname{VAF} \hat{K} / \hat{G}$ AND $\hat{K} / \hat{B} / \hat{I} / \hat{G}$, VARIabiLITY, VAR $\hat{K} / \hat{G}$, AND RISETIME, $\tau_{K} / \tau_{G}$ OF THE IDENTIFIED PaRAmEters

\begin{tabular}{|c|c|c|c|c|c|c|c|}
\hline & Original [9] & $\mathrm{OO}$ & OC & $\mathrm{CO}$ & $\mathrm{CC}$ & $\mathrm{CEO}$ & CECE \\
\hline VAF $\hat{K}[\%]$ & 98.6 & 99.0 & 99.2 & 98.4 & 98.6 & 98.6 & 98.7 \\
\hline$\hat{G}[\mathrm{Nm} / \mathrm{rad} / \mathrm{s}](G=10)$ & $10.2 \pm 0.5$ & $10.3 \pm 0.4$ & $10.4 \pm 0.4$ & $10.6 \pm 0.7$ & $10.2 \pm 0.7$ & $12.3 \pm 0.8$ & $10.3 \pm 0.7$ \\
\hline VAF $\hat{G}[\%]$ & 99.3 & 99.7 & 99.6 & 99.1 & 99.0 & 99.5 & 99.0 \\
\hline$\hat{K}[\mathrm{Nm} / \mathrm{rad}](K=100)$ & $98 \pm 5$ & $96 \pm 4$ & $97 \pm 4$ & $94 \pm 5$ & $97 \pm 5$ & $85 \pm 5$ & $97 \pm 5$ \\
\hline$\hat{B}[\mathrm{Nm} / \mathrm{rad} / \mathrm{s}](B=0.63)$ & $0.63 \pm 0.03$ & $0.63 \pm 0.04$ & $0.95 \pm 0.03$ & $0.50 \pm 0.03$ & $0.66 \pm 0.02$ & $0.58 \pm 0.02$ & $0.66 \pm 0.02$ \\
\hline$\hat{I}\left[\mathrm{Nm} / \mathrm{rad} / \mathrm{s}^{2}\right](I=0.0137)$ & $0.0137 \pm 2 \mathrm{e}-4$ & $0.0136 \pm 3 e-4$ & $0.0130 \pm 4 \mathrm{e}-4$ & $0.0072 \pm 2 \mathrm{e}-4$ & $0.0135 \pm 4 \mathrm{e}-4$ & $0.0001 \pm 9 \mathrm{e}-6$ & $0.0135 \pm 4 \mathrm{e}-4$ \\
\hline $\operatorname{var} \hat{K}[\%$ of mean] & 7.9 & 6.7 & 7.6 & 8.4 & 9.4 & 9.0 & 9.4 \\
\hline $\operatorname{var} \hat{G}[\%$ of mean $]$ & 5.2 & 3.0 & 4.0 & 5.1 & 6.8 & 4.6 & 7.2 \\
\hline$\tau_{K}[\mathrm{~s}]$ & 14.2 & 14.3 & 15.1 & 13.9 & 16.1 & 56.3 & 16.2 \\
\hline$\tau_{G}[\mathrm{~s}]$ & 11.9 & 9.5 & 8.5 & 9.4 & 8.4 & 7.7 & 8.3 \\
\hline
\end{tabular}
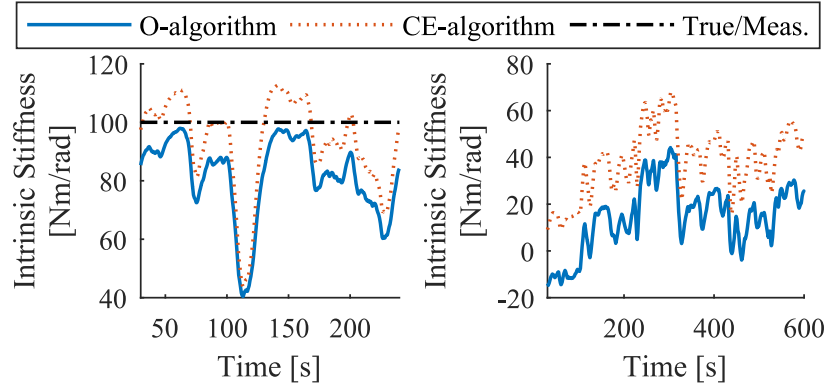

Fig. 6. O- and CE-algorithm parameter identification performance for the (Left) Simulation (CE-model condition) and (Right) Experimental study
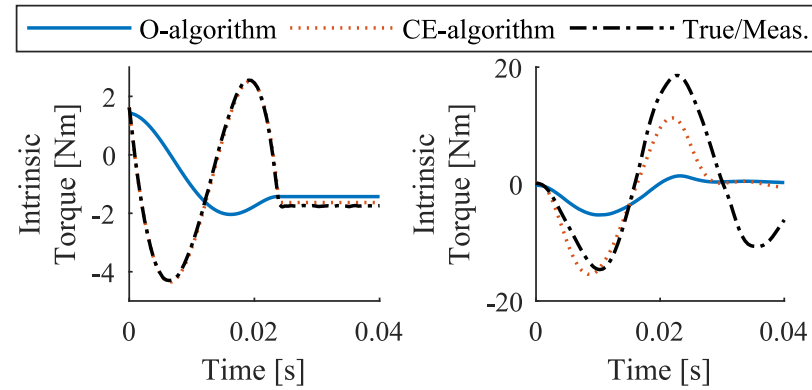

Fig. 7. O- and CE-algorithm intrinsic torque \%VAF performance after step perturbation for the (Left) Simulation (CE-model condition) and (Right) Experimental study

closer to simulated truth and measured reality compared with the O-algorithm for the identified intrinsic stiffness, Fig. 6, and intrinsic torque, Fig. 7. This is confirmed by the \% VAF for the intrinsic torque of $99.6 \%$ for a training and validation set for the CE-algorithm versus $-32.1 \%$ and $-33.3 \%$ for the O-algorithm. Additionally, the experimental \% VAF for intrinsic torque is $66.2 \%$ versus $26.6 \%$ for the CEand O-algorithms respectively. Fig. 7 shows that part of this low VAF is cause by the elongated torque response measured during experiments, which is not seen in simulation.

\section{Validation of Identification Algorithm}

Given its superior performance, the CE-algorithm is used to validate the estimates of the intrinsic and reflexive stiffness with EMG measurements. Fig. 8 shows a concurrent increase for both intrinsic stiffness and background activity, and
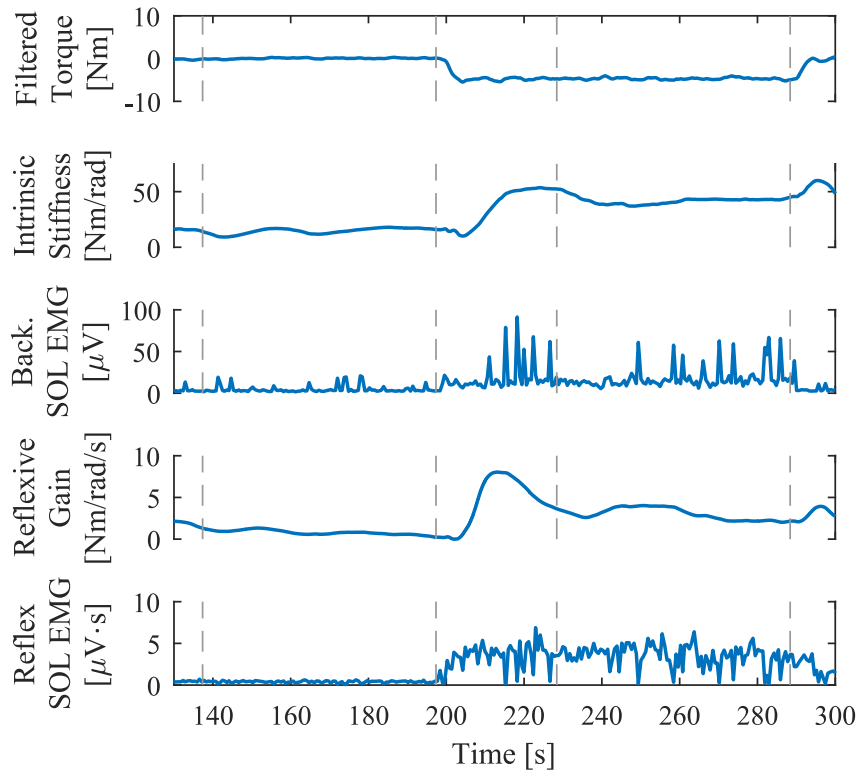

Fig. 8. Timeseries of low-pass filtered torque, identification and EMG parameters for two $60 \mathrm{~s}$ hold periods, indicated with the grey dotted lines

reflexive gain and reflex activity in the Soleus muscle.

The consistency of these observed relationships over all hold periods and subjects for the four muscles and two torque targets are shown in Table III. These results show that there is a large linear association, up to $86 \%$ shared variance with a lower confidence bound of $31 \%$, between the intrinsic stiffness and background EMG activity for all four muscles at the $0 \mathrm{Nm}$ torque target. Similarly, the three calf muscles (SOL, GL, GM) also have a large linear association, up to $67 \%$ shared variance with a lower confidence bound of $34 \%$ between the reflexive gain and reflex EMG activity at the $0 \mathrm{Nm}$ torque target. The same positive correlations, for both pathways, were found at the $-5 \mathrm{Nm}$ torque target, although the linear association is clearly weaker. The correlation for the reflexive TA activity is weaker for the $0 \mathrm{Nm}$ target and non-existent at the $-5 \mathrm{Nm}$ target, given the negative lower confidence bound. Note, the intrinsic properties of the GL muscle at $-5 \mathrm{Nm}$ torque showed unexpected behavior, i.e. a small correlation and a negative confidence bound, compared to all other conditions, most likely due to outliers. 
TABLE III

PEARSON'S CORRELATION COEFFICIENTS ( $r$ ) AND THEIR 95\% CONFIDENCE INTERVALS BETWEEN IDENTIFIED INTRINSIC STIFFNESS AND BACKGROUND EMG ACTIVITY (INTRINSIC) AND IDENTIFIED REFLEXIVE GAIN AND REFLEX EMG ACTIVITY (REFLEXIVE)

\begin{tabular}{l|cc|cc} 
& \multicolumn{2}{|c|}{ Intrinsic } & \multicolumn{2}{c}{ Reflexive } \\
Torque & $0 \mathrm{Nm}(N=33)$ & $-5 \mathrm{Nm}(N=22)$ & $0 \mathrm{Nm}(N=33)$ & $-5 \mathrm{Nm}(N=22)$ \\
\hline SOL & $0.93[0.85,0.97]$ & $0.70[0.44,0.87]$ & $0.78[0.62,0.87]$ & $0.57[0.20,0.77]$ \\
TA & $0.88[0.78,0.95]$ & $0.70[0.19,0.87]$ & $0.55[0.27,0.75]$ & $0.18[-0.11,0.45]$ \\
GL & $0.91[0.83,0.96]$ & $0.36[-0.18,0.69]$ & $0.75[0.58,0.85]$ & $0.56[0.21,0.76]$ \\
GM & $0.80[0.56,0.92]$ & $0.65[0.32,0.82]$ & $0.82[0.67,0.90]$ & $0.46[0.13,0.67]$
\end{tabular}

\section{DisCuSSION AND CONCLUSION}

The aim of this study was to validate the online estimates of intrinsic and reflexive joint impedance using EMG measurements in co-contraction conditions. Both the intrinsic and reflexive pathways show a large linear association between the system identification and EMG measures for the calf muscles at zero net torque. At a small plantarflexion torque, this positive association also exists although it is weaker, especially for the reflexive pathway. As the algorithm estimates are focused on the calf muscles, because half-wave rectified velocity is used, a lower association is also seen for the reflexive pathway of the TA muscle.

The validated system identification method includes incremental improvements on top of [9]. The modifications do not specifically consider spastic participants, as similar research has shown that the combination of a second-order intrinsic and non-parametric reflexive model should suit spastic participants equally well [13]. The results show that reshaping the perturbation signal is needed for systems with a limited bandwidth to comply with the stated requirements [9]. Moreover, using actual velocity and acceleration, a variable-step solver and anti-aliasing filters in the model creates a better match with reality. However, these changes introduce differences between the modified model and original algorithm, reducing the quality of identified parameters due to increased bias.

An improved algorithm, using higher-order backwards difference approximators and $100 \mathrm{~Hz}$ low-pass filters on the algorithm inputs, mitigates the induced parameter bias, for both simulation and experiment. The $100 \mathrm{~Hz}$ low-pass filters, in combination with direct velocity measurements, also help to properly mitigate increased bias due to reduced SNR. Besides, the general applicability of the algorithm in other programming languages is improved by using digital instead of analog filters.

Despite the modifications, limited actuator performance of the specific experimental setup used did increase variability of the identified parameters. This increase could potentially impede a training paradigm based on the algorithm as clarity of the biofeedback is reduced. Besides, the simulation and experimental results show a large difference in \%VAF. This is partially explained by an elongated torque response in the intrinsic time frame during the experiments. This elongated, oscillatory torque response continues into the reflexive time frame and will thus also affect the reflexive gain identification. To reduce this elongated torque response, the control scheme of the actuator should be revised. Another discrepancy between simulation and experiment is the sim- plified 2nd-order model used for the intrinsic pathway, while evidence exists that a more complex model is required [14].

The most important result of the study is that the used system identification technique has been validated using independently measured and processed EMG measures. In general, this result shows the validity of the used system identification technique to study a human physiological system. More specifically, the successful validation of the algorithm shows that the method has the potential to be used as training paradigm to reduce hyperreflexia in the future.

\section{REFERENCES}

[1] J. W. Lance, "Symposium Synopsis," in Spasticity: Disordered Motor Control, R. G. Feldman, R. R. Young, and W. P. Koella, Eds. Chicago, IL, USA: Year Book Medical Publishers, 1980, pp. 485-495.

[2] V. Dietz and T. Sinkjaer, "Spastic Movement Disorder: Impaired Reflex Function and Altered Muscle Mechanics," Lancet Neurology, vol. 6 , no. 8, pp. 725-733, 2007.

[3] J. A. Burne, V. L. Carleton, and N. J. O'Dwyer, "The Spasticity Paradox: Movement Disorder or Disorder of Resting Limbs?” Journal of Neurology, Neurosurgery and Psychiatry, vol. 76, no. 1, pp. 47-54, 2005.

[4] A. K. Thompson, F. R. Pomerantz, and J. R. Wolpaw, "Operant Conditioning of a Spinal Reflex Can Improve Locomotion after Spinal Cord Injury in Humans," Journal of Neuroscience, vol. 33, no. 6, pp. 2365-2375, 2013.

[5] R. L. Segal and S. L. Wolf, "Operant Conditioning of Spinal Stretch Reflexes in Patients with Spinal Cord Injuries," Experimental Neurology, vol. 130, no. 2, pp. 202-213, 1994.

[6] D. Ludvig, I. Cathers, and R. E. Kearney, "Voluntary Modulation of Human Stretch Reflexes," Experimental Brain Research, vol. 183, no. 2, pp. 201-213, 2007.

[7] R. E. Kearney, R. B. Stein, and L. Parameswaran, "Identification of Intrinsic and Reflex Contributions to Human Ankle Stiffness Dynamics," IEEE Transactions on Biomedical Engineering, vol. 44, no. 6, pp. 493-504, 1997.

[8] R. L. Segal, "Spinal Cord Plasticity is a Possible Tool for Rehabilitation," Neurology Report, vol. 22, no. 2, pp. 54-60, 1998.

[9] D. Ludvig and R. E. Kearney, "Real-time Estimation of Intrinsic and Reflex Stiffness," IEEE Transactions on Biomedical Engineering, vol. 54, no. 10, pp. 1875-1884, 2007.

[10] H. J. Hermens, B. Freriks, C. Disselhorst-Klug, and G. Rau, "Development of Recommendations for SEMG Sensors and Sensor Placement Procedures," Journal of Electromyography and Kinesiology, vol. 10, no. 5, pp. 361-374, 2000.

[11] J. Carpenter and J. Bithell, "Bootstrap Confidence Intervals: When, Which, What? A Practical Guide for Medical Statisticians," Statistics in Medicine, vol. 19, no. 9, pp. 1141-1164, 2000.

[12] R Core Team, R: A Language and Environment for Statistical Computing, R Foundation for Statistical Computing, Vienna, Austria, 2018. [Online]. Available: http://www.R-project.org/

[13] M. M. Mirbagheri, H. Barbeau, M. Ladouceur, and R. E. Kearney, "Intrinsic and Reflex Stiffness in Normal and Spastic, Spinal Cord Injured Subjects," Experimental Brain Research, vol. 141, no. 4, pp. 446-459, 2001.

[14] E. Sobhani Tehrani, K. Jalaleddini, and R. E. Kearney, "Ankle Joint Intrinsic Dynamics is More Complex than a Mass-Spring-Damper Model," IEEE Transactions on Neural Systems and Rehabilitation Engineering, vol. 25, no. 9, pp. 1568-1580, 2017. 